\title{
On relativistic models in the equilibrium statistical mechanics
}

\author{
V.Tretyak \\ Institute for Condensed Matter Physics of the Ukrainian National Academy \\ of Sciences, 1 Svientsitskii St., UA-290011 Lviv-11, Ukraine
}

Received June 12, 1998

Relativistic effects in the thermodynamical properties of interacting particle systems are investigated within the framework of the relativistic direct interaction theory in various forms of dynamics. In the front form of relativistic dynamics an exactly solvable model of a one-dimensional hard spheres gas is formulated and an equation of state and thermodynamical potentials for such a gas are found. Weakly relativistic corrections to the thermodynamical functions of the dilute gas with short-range interactions are discussed on the basis of the approximately relativistic Hamiltonian function in the instant form of dynamics.

Key words: relativistic statistical mechanics, forms of dynamics, relativistic ideal gas, weakly relativistic corrections

PACS: $05.70 . \mathrm{C}$

\section{Introduction}

The present status of the relativistic direct interaction theory [1-4] enables us to consider it as a natural basis for a consistent description of relativistic effects in various physical systems on different levels: classical, quantum, or statistical ones. The relativistic statistical mechanics of interacting particle systems is nowadays at an early stage of its development, although the classical partition function of a relativistic ideal gas was calculated by Jüttner in 1911. One cause of this was indicated in report [5]. Ter Haar and Wergeland wrote: "At extremely high temperatures relativistic effects may, of course, be important. Then, however, matter behaves as a mixture of ideal gases and this limiting case poses no problem. By and large, a relativistic theory of heat seems, therefore, to be of little practical importance". But the 'practical importance', being a very non-smooth function on historical time, cannot be considered as the main reason for theoretical investigation.

Among various approaches to the relativistic direct interaction theory the single-time Lagrangian formalism $[1,6,7]$ seems to be the most convenient in the 
consideration of the general problem of relativistic dynamics, as well as in the investigation of various approximations. This formalism has been extended to an arbitrary form of relativistic dynamics defined geometrically by means of space-like foliations of the Minkowski space [8,9]. Transition from the classical Lagrangian to the Hamiltonian description allows one to consider the relativistic effects in the statistical and quantum-mechanical properties of the particle systems.

The present paper is concerned with relativistic models of the equilibrium statistical mechanics. In section 2 , a brief introduction into the concept of the form of relativistic dynamics is presented. Investigation of a classical and quantum relativistic ideal gas by means of the front form of dynamics is outlined in section 3 . Relativistic generalization of a one-dimensional gas of hard spheres is obtained in section 4. This gives an example of an exactly solvable model in the relativistic statistical mechanics with a non-trivial particle interaction. Section 5 is devoted to the investigation of the first quasirelativistic (post-Newtonian) approximation. In the instant form of dynamics the weakly relativistic corrections to the thermodynamical functions of the dilute gas with short-range interactions are studied on the basis of the general structure, to the order $c^{-2}$, of the approximately relativistic Hamiltonian. As an example, the relativistic correction to the Van der Waals equation to the order $c^{-2}$ is obtained.

\section{Forms of relativistic dynamics in the Lagrangian description of an interacting particle system}

Let us consider a dynamical system consisting of $N$ interacting point particles. It is convenient to describe the evolution of this system in the $(n+1)$-dimensional Minkowski space $\mathbb{M}_{n+1}$ with coordinates $x^{\mu}, \mu=0,1, \cdots, n$. We use the metric $\left\|\eta_{\mu \nu}\right\|=\operatorname{diag}(1, \underbrace{-1, \cdots,-1}_{n})$. In applications we put $n=3$ or $n=1$. The motion of the particles is described by the world lines

$$
\gamma_{a}: \mathbb{R} \rightarrow \mathbb{M}_{n+1}, \quad \tau_{a} \mapsto x_{a}^{\mu}\left(\tau_{a}\right) ; \quad a=1, \ldots, N,
$$

being time-like one-dimensional unbounded submanifolds of the Minkowski space.

The relativistic freedom in the simultaneity definition makes possible different three-dimensional descriptions of the relativistic particle motions. According to Dirac $[10,11]$, they are called the forms of relativistic dynamics. Within the framework of the single-time Lagrangian or Hamiltonian mechanics this concept may be introduced in the following way $[8,7]$. Let us consider foliation $\Sigma$ of the Minkowski space $\mathbb{M}_{n+1}$ by the hypersurfaces

$$
t=\sigma(x), \quad t \in \mathbb{R},
$$

with the next property: every hypersurface $\Sigma_{t}=\left\{x \in \mathbb{M}_{n+1} \mid \sigma(x)=t\right\}$ must intersect the world lines $\gamma_{a}$ of all the particles in one and only one point

$$
x_{a}(t)=\gamma_{a} \bigcap \Sigma_{t}
$$


This allows us to consider $t$ as an evolution parameter of the system [8,11]. In the Poincaré-invariant theory, when we consider only time-like world lines, the hypersurfaces (2.2) must be space-like or isotropic:

$$
\eta_{\mu \nu}\left(\partial^{\mu} \sigma\right)\left(\partial^{\nu} \sigma\right) \geq 0
$$

where $\partial^{\mu}=\partial / \partial x_{\mu}$. Then, we have $\partial^{0} \sigma>0$, and the hypersurface equation (2.2) has the solution $x^{0}=\varphi(t, \mathbf{x})$, where $\mathbf{x}=\left(x^{i}\right), i=1, \cdots, n$. Therefore, the constraint $x_{a}(t) \in \Sigma_{t}$ enables us to determine the zeroth component of $x_{a}(t)$ in terms of $t$ and $x_{a}^{i}(t)$. The parametric equations (2.1) of the world lines of the particles in the given form of dynamics have the form:

$$
x^{0}=\varphi\left(t, \mathbf{x}_{a}(t)\right) \equiv \varphi_{a}, \quad x^{i}=x_{a}^{i}(t) .
$$

The evolution of the system is determined by $n N$ functions $t \mapsto x_{a}^{i}(t)$. They may be considered as representatives for the sections $s: \mathbb{R} \rightarrow \mathbb{F}, t \mapsto\left(t, x_{a}^{i}(t)\right)$ of the trivial fibre bundle $\pi: \mathbb{F} \rightarrow \mathbb{R}$ with $n N$-dimensional fibre space $\mathbb{E}=\mathbb{R}^{n N}$. The latter constitutes the configuration space of our system.

Three Dirac's forms of relativistic dynamics correspond to the following hypersurfaces (2.2): $x^{0}=c t$ (instant form), $x^{0}+x^{n}=c t$ (front form), and $\eta_{\mu \nu} x^{\mu} x^{\nu}=c^{2} t^{2}$ (point form). Other examples for the case $n=3$ may be found in [8].

In the relativistic Lagrangian mechanics the Lagrangian function $L: J^{\infty} \pi \rightarrow \mathbb{R}$ is defined on the infinite order jet space of the fibre bundle $\pi: \mathbb{F} \rightarrow \mathbb{R}$ with the standard coordinates $x_{a}^{i(s)}$ [12]. The values of these coordinates for the section $s: t \mapsto\left(t, x_{a}^{i}(t)\right)$ belonging to the corresponding equivalence class from $J^{\infty} \pi$ are $x_{a}^{i(s)}(t)=\mathrm{d}^{s} x_{a}^{i}(t) / \mathrm{d} t^{s} \equiv D^{s} x_{a}^{i}, s=0,1,2, \cdots$.

The free-particle system is determined in any form of dynamics by the Lagrangian $L_{f}: J^{1} \pi \rightarrow \mathbb{R}$ depending on the first derivatives:

$$
L_{f}=\sum_{a} m_{a} c \sqrt{(D \varphi(t, \mathbf{x}))^{2}-v_{a}^{2}} ; \quad v_{a}^{i} \equiv x_{a}^{i(1)},
$$

$m_{a}$ being a rest mass of the particle. In the front form of dynamics it reads:

$$
L_{f}=\sum_{a} m_{a} c^{2} \sqrt{1-\left(v_{a 1}^{2}+\cdots+v_{a(n-1)}^{2}\right) / c^{2}-2 v_{a n} / c} .
$$

In the general case the Poincaré-invariance conditions forbid the existence of interaction Lagrangians which are defined on the jet-space $J^{r} \pi$ with some finite $r$ (for example, with $r=1$ ). This leads to serious difficulties in physical interpretation of the formalism, and, in fact, makes it impossible to obtain a closed form of the corresponding Hamiltonian functions which is necessary for the development of the classical statistical mechanics $[1,7,8]$. This fact is the Lagrangian counterpart of the famous no-interaction theorem in the Hamiltonian relativistic mechanics [13].

There are at least two possibilities to avoid this difficulty. The first is offered by the front form of dynamics in the two-dimensional Minkowski space. In this case 
there exists a wide class of interaction Lagrangians depending on the first order derivatives [14]. This allows us to obtain corresponding Hamiltonian functions by the standard Legendre transformation that preserves the physical meaning of the position canonical coordinates. The second consists in the consideration of the approximation in $c^{-2}[1,9,15]$. At least in the first order approximation we may obtain the usual Lagrangian functions depending on the first order derivatives [1]. Examples of using these possibilities in the investigation of relativistic effects in models of the equilibrium statistical mechanics will be considered in the next sections.

\section{Relativistic ideal gas within the framework of the front form of dynamics}

As an illustrative example we shall consider here a description of the relativistic ideal gas by means of the front form of dynamics. The Hamiltonian function of the free $N$-particle system has the form:

$$
H=\sum_{a=1}^{N} H_{a}\left(\mathbf{x}_{a}, \mathbf{p}_{a}\right)
$$

with

$$
H_{a}=H_{0}(\mathbf{p})=c \frac{m^{2} c^{2}+\mathbf{p}_{a}^{2}}{2 p_{a 3}} .
$$

This Hamiltonian can be obtained from the Lagrangian (2.7) by the usual Legendre application.

The $n$-dimensional coordinates $\mathbf{x}_{a}$ cover the given region $\Omega \subset \mathbb{R}^{n}$ with $\operatorname{vol}(\Omega)=$ $V$, and $\mathbf{p}_{a}$ belongs to the region $\Pi=\left\{\mathbf{p}_{a}=\left(p_{a 1}, \ldots, p_{a n}\right) \in \mathbb{R}^{n} \mid p_{a n}>0\right\}$. As it is well known, all the information about thermodynamical properties of the system is contained in the expression for the canonical partition function [16]:

$$
Z_{N}=\frac{1}{h^{n N} N !} \int \mathrm{e}^{-\beta H} \prod_{a} \mathrm{~d}^{n} x_{a} \mathrm{~d}^{n} p_{a},
$$

where, as usual, $\beta=(k T)^{-1}$ and the integration is performed over the phase space $\mathbb{P}$. In our case $\mathbb{P}=\Omega^{N} \times \Pi^{N}$. Inserting (3.1) into equation (3.3) we obtain

$$
Z_{N}=\frac{1}{h^{n N} N !} z^{N}
$$

where

$$
z=\int \mathrm{e}^{-\beta H_{a}} \mathrm{~d}^{n} x_{a} \mathrm{~d}^{n} p_{a} .
$$

Substitution of the front form Hamiltonian (3.2) immediately yields

$$
z=V \int_{0}^{\infty} \mathrm{d} p_{n} \exp \left(-\frac{1}{2} \beta c\left(p_{n}+\frac{m^{2} c^{2}}{p_{n}}\right)\right) \prod_{k=1}^{n-1} \int_{-\infty}^{\infty} \mathrm{d} p_{k} \exp \left(-\frac{\beta c}{2 p_{n}} p_{k}^{2}\right) .
$$


Performing integration over $p_{k}$ and putting $p_{n}=m c \alpha$ we have

$$
z=V m c\left(\frac{2 \pi m}{\beta}\right)^{(n-1) / 2} \int_{0}^{\infty} \mathrm{d} \alpha \alpha^{(n-1) / 2} \exp \left(-\frac{\beta m c^{2}}{2}\left(\alpha+\frac{1}{\alpha}\right)\right) .
$$

Using the integral representation of the Hankel function $K_{n}(x)=\frac{\pi \mathrm{i}}{2} \mathrm{e}^{i n \pi / 2} H_{n}^{(1)}(\mathrm{i} x)$,

$$
K_{n}(x)=\frac{1}{2} \int_{0}^{\infty} \mathrm{d} \alpha \alpha^{n-1} \exp \left(-\frac{x}{2}\left(\alpha+\frac{1}{\alpha}\right)\right), \quad x>0,
$$

we obtain

$$
z=2 V m c\left(\frac{2 \pi m}{\beta}\right)^{(n-1) / 2} K_{(n+1) / 2}\left(\beta m c^{2}\right) .
$$

When $n=3$, equations (3.4) and (3.9) give well-known Jüttner's result:

$$
Z_{N}=\frac{1}{N !}\left[\frac{4 \pi m^{2} c V}{\beta h^{3}} K_{2}\left(\beta m c^{2}\right)\right]^{N},
$$

which is usually derived by means of the instant form Hamiltonian:

$$
H_{a}=c \sqrt{m^{2} c^{2}+\mathbf{p}_{a}^{2}}
$$

Using the asymptotical expansion

$$
K_{n}(x) \asymp \sqrt{\frac{\pi}{2 x}} \mathrm{e}^{-x}\left(1+\frac{4 n^{2}-1}{8 x}+\ldots\right), \quad x \rightarrow \infty,
$$

we can obtain a weakly relativistic correction to the non-relativistic result:

$$
Z_{N}=Z_{N}^{(0)} \mathrm{e}^{-\beta N m c^{2}}\left(1+\frac{n(n+2)}{8 \beta m c^{2}}\right)^{N},
$$

where

$$
Z_{N}^{(0) i d}=\frac{V \Lambda^{-n N}}{N !}, \quad \Lambda \equiv \sqrt{\frac{\beta h^{2}}{2 \pi m}} .
$$

The necessity for (rather trivial) renormalization of the non-relativistic partition function $Z_{N}^{(0)} \rightarrow Z_{N}^{(0)} \mathrm{e}^{-\beta N m c^{2}}$ follows from the presence of the rest energy $m c^{2}$ in the relativistic Hamiltonians (3.2) or (3.11).

The obtained expressions can be useful for the treatment of the quantum relativistic ideal gas with generalized statistics of arbitrary order $q$. In this case it is convenient to consider the grand partition function

$$
\Theta=\operatorname{Tre}^{-\beta(\mathrm{H}-\mu \mathrm{N})} \equiv \mathrm{e}^{-\beta \Omega},
$$

where $\mathrm{H}$ and $\mathrm{N}$ represent the operators of energy and of particle number, respectively, and $\mu$ denotes chemical potential [16]. 
Assuming that the maximum value of the occupation number in a given energy state can be $q$, any integer greater than 1 , and acting in a manner quite similar to the non-relativistic case (cf., for example, [17]), we obtain:

$$
\Omega=-\frac{g V}{\beta h^{n}} \int \mathrm{d}^{n} p \ln \left(\frac{1-\exp \left(-\beta(q+1)\left(H_{0}(\mathbf{p})-\mu\right)\right)}{1-\exp \left(-\beta\left(H_{0}(\mathbf{p})-\mu\right)\right)}\right) .
$$

Here $g$ denotes degeneracy of the energy state with a given value of momentum $\mathbf{p}$. For structureless particles with the spin $\sigma$ we have $g=2 \sigma+1$.

Of course, care is needed in the replacement of the summation by the integration over the momentum states, when $q \sim N$, and, especially, if $q \rightarrow \infty$ (that corresponds to the Bose-Einstein statistics). But this problem, connected with the Bose condensation, is essential at a low temperature, $\beta^{-1}<<m c^{2}$, when relativistic correction seems to be negligible. Then the standard thermodynamical relations lead to the following expression for the pressure:

$$
P=g \beta^{-1} h^{-n} \int d^{n} p\left[\ln \left(1-\lambda^{q+1} \mathrm{e}^{-\beta(q+1) H_{0}(\mathbf{p})}\right)-\ln \left(1-\lambda \mathrm{e}^{-\beta H_{0}(\mathbf{p})}\right)\right],
$$

where

$$
\lambda=\mathrm{e}^{\beta \mu}
$$

is the fugacity. The number density

$$
\frac{1}{v}=\frac{N}{V}=\frac{\partial P}{\partial \mu}
$$

is given by

$$
\frac{1}{v}=g h^{-n} \int \mathrm{d}^{n} p\left[\frac{\lambda \exp \left(-\beta H_{0}(\mathbf{p})\right)}{1-\lambda \exp \left(-\beta H_{0}(\mathbf{p})\right)}-(q+1) \frac{\lambda^{q+1} \exp \left(-\beta(q+1) H_{0}(\mathbf{p})\right)}{1-\lambda^{q+1} \exp \left(-\beta(q+1) H_{0}(\mathbf{p})\right)}\right]
$$

and the inner energy density $u=U / V$ is found to be

$u=g h^{-n} \int \mathrm{d}^{n} p H_{0}(\mathbf{p})\left[\frac{\lambda \exp \left(-\beta H_{0}(\mathbf{p})\right)}{1-\lambda \exp \left(-\beta H_{0}(\mathbf{p})\right)}-\frac{(q+1) \lambda^{q+1} \exp \left(-\beta(q+1) H_{0}(\mathbf{p})\right)}{1-\lambda^{q+1} \exp \left(-\beta(q+1) H_{0}(\mathbf{p})\right)}\right]$.

Expanding the exponential functions into the series and using the denotation

$$
\phi(\beta)=g h^{-n} z / V
$$

where $z$ is defined by equation (3.5), we have

$$
\beta P=\sum_{k=1}^{\infty} k^{-1}\left[\lambda^{k} \phi(\beta k)-\lambda^{k(q+1)} \phi(\beta(q+1) k)\right]
$$

and

$$
\frac{1}{v}=\sum_{k=1}^{\infty}\left[\lambda^{k} \phi(\beta k)-(q+1) \lambda^{k(q+1)} \phi(\beta(q+1) k)\right] .
$$


Keeping only the terms up to the second order in the density $1 / v$, we obtain an equation of state of the form:

$$
\beta P=\frac{1}{v}-\frac{1}{2 v^{2}} \eta_{q} \frac{\phi(2 \beta)}{\phi(\beta)},
$$

where

$$
\eta_{q}=\left\{\begin{array}{cc}
-1, & q=1 \\
1, & q>1
\end{array}\right.
$$

Similarly, for the energy density with the same accuracy we have

$$
u=-\frac{1}{v} \frac{\phi^{\prime}(\beta)}{\phi(\beta)}+\frac{1}{2 v^{2}} \eta_{q} \frac{\phi(2 \beta) \phi^{\prime}(\beta)-\phi(\beta) \phi^{\prime}(2 \beta)}{\phi(\beta)^{3}} .
$$

Making use of the explicit expression for the $\phi$ function from (3.9) and (3.22), we may find a relativistic equation of state at a high temperature up to the first order in the degeneracy parameter

$$
\delta=\frac{\Lambda^{n}}{g v}
$$

The equation is found to be

$$
\beta P=\frac{1}{v}\left[1-\frac{\eta_{q} \delta}{2^{1+n / 2}} F\left(\beta m c^{2}\right)\right],
$$

where

$$
F(x)=\sqrt{\frac{\pi}{x}} \frac{K_{(n+1) / 2}(2 x)}{K_{(n+1) / 2}^{2}(x)} .
$$

Because $F(x) \rightarrow 1$ as $x \rightarrow \infty$, equation (3.29) agrees with the known nonrelativistic results [16]. In a similar manner we may write relativistic virial expansions for other thermodynamical functions of the quantum ideal gas, especially for the inner energy and specific heat.

\section{Relativistic one-dimensional model of the hard spheres gas}

In the two-dimensional space-time $\mathbb{M}_{2}$, the front form of dynamics serves a wide class of non-trivial interaction Hamiltonians in terms of the covariant canonical coordinates.

The general form of a Hamiltonian function for a system of $N$ identical particles on the line $\mathbb{I}=\{x \in \mathbb{R} \mid 0<x<A\}$ described by the canonical coordinates $x_{a}$ and momenta $p_{a}$ within the framework of the front form of dynamics is given by [10]:

$$
H=\sum_{a} H_{0}\left(p_{a}\right)+\sum_{a<b} \sum_{a}\left(p_{a}+p_{b}\right) V\left(r_{a b} p_{a}, r_{a b} p_{b}\right)
$$


Here

$$
H_{0}(p)=\frac{1}{2}\left(p+\frac{m^{2}}{p}\right)
$$

$r_{a b} \equiv x_{a}-x_{b}$, and $\mathrm{V}$ is an arbitrary function on the indicated arguments. In this section we put $c=1$. From the definition of the front form of dynamics it follows that momenta $p_{a}$ belong to the positive semiaxis $\mathbb{R}_{+}=\{x \in \mathbb{R} \mid x>0\}$ :

$$
p_{a}>0 .
$$

For the convenience of the comparison with the corresponding non-relativistic calculations we assume that function $V$ has the form:

$$
V=V\left(\left|q_{a b}\right|\right)
$$

where

$$
q_{a b}=r_{a b}\left(p_{a}+p_{b}\right) \nu\left(\frac{p_{a}}{p_{b}}+\frac{p_{b}}{p_{a}}\right)
$$

and $\nu: \mathbb{R}_{+} \rightarrow \mathbb{R}_{+}$is some function which will be defined later.

Let us again consider the canonical partition function

$$
Z_{N}=\frac{1}{h^{N} N !} \int \mathrm{e}^{-\beta H} \prod_{a} \mathrm{~d} x_{a} \mathrm{~d} p_{a},
$$

over the phase space $\mathbb{P}=\mathbb{I}^{N} \times \mathbb{R}_{+}^{N}$.

Since we are interested in the relativistic generalization of the hard spheres model, we can choose interaction function (4.4) in the form

$$
V(x)=\left\{\begin{array}{cc}
0, & x>\sigma \\
\infty, & x<\sigma
\end{array}\right.
$$

We, therefore, find

$$
\mathrm{e}^{-\beta V\left(\left|q_{a b}\right|\right)}= \begin{cases}1, & \left|q_{a b}\right|>\sigma \\ 0, & \left|q_{a b}\right|<\sigma\end{cases}
$$

If we choose function $\nu(x)$ in such a way that for any $a, b, c$

$$
\text { if }\left|q_{a b}\right|>\sigma \text { and }\left|q_{b c}\right|>\sigma \text {, then }\left|q_{a c}\right|>\sigma,
$$

the partition function (4.6) can be written in the form:

$$
Z_{N}=\frac{1}{h^{N} N !} \int \prod_{a} \mathrm{~d} \mu\left(p_{a}\right) \int \prod_{a} \mathrm{~d} x_{a},
$$

where

$$
\mathrm{d} \mu(p)=\mathrm{e}^{-\beta H_{0}(p)} \mathrm{d} p
$$


and coordinates $x_{a}$ must satisfy the restrictions

$$
\left|r_{a b}\right|>\frac{\sigma}{p_{a}+p_{b}} u\left(\frac{p_{a}}{p_{b}}+\frac{p_{b}}{p_{a}}\right), \quad u(x) \equiv \frac{1}{\nu(x)} .
$$

Conditions (4.9) lead to the inequality

$$
\frac{x}{4} \leqslant \frac{u(x)}{u(2)} \leqslant \frac{x+2}{4}
$$

In the following we shall choose the simplest solution of (4.13) which has the form

$$
u(x)=C(x+2),
$$

such that

$$
q_{a b}=C^{-1} r_{a b}\left(\frac{1}{p_{a}}+\frac{1}{p_{b}}\right)^{-1} .
$$

Because in the non-relativistic limit $p_{a} \rightarrow m$, the demand that $q_{a b} \rightarrow r_{a b}$ in this limit fixes the value of the constant $C$ :

$$
C=\frac{m}{2}
$$

Therefore, partition function (4.10) may be rewritten in the form:

$$
Z_{N}=\frac{1}{h^{N}} \int\left(\prod_{a=1}^{N} \mathrm{~d} \mu\left(p_{a}\right) \mathrm{d} x_{a}\right) \prod_{a=1}^{N-1} \theta\left(x_{a+1}-x_{a}-\frac{m \sigma}{2 p_{a}}-\frac{m \sigma}{2 p_{a+1}}\right) .
$$

The appearance of Heaviside $\theta$ functions is a consequence of conditions (4.12). Let us perform in (4.17) a change of the variables $\left(x_{a}, p_{a}\right) \rightarrow\left(y_{a}, p_{a}\right)$, such that the arguments of $\theta$ functions become $y_{a+1}-y_{a}$. This gives

$$
y_{a}=x_{a}-\frac{m \sigma}{2} \varphi_{a}
$$

where

$$
\varphi_{1}=0, \quad \varphi_{2}=\frac{1}{p_{1}}+\frac{1}{p_{2}}
$$

and

$$
\varphi_{a}=\frac{1}{p_{1}}+2 \sum_{b=2}^{a-1} \frac{1}{p_{b}}+\frac{1}{p_{a}}, \quad a>2 .
$$

As a consequence, we obtain

$$
Z_{N}(A, \beta)=\frac{1}{h^{N} N !} \int \prod_{a=1}^{N} \mathrm{~d} \mu\left(p_{a}\right) \theta\left(A-\frac{m \sigma}{2} \varphi_{N}\right)\left(A-\frac{m \sigma}{2} \varphi_{N}\right)^{N} .
$$


Before performing an integration over momenta we consider the Laplace transformation of (4.21)

$$
\begin{aligned}
Z_{N}(s, \beta) & =\int_{0}^{\infty} \mathrm{d} A \mathrm{e}^{-s A} Z_{N}(A, \beta) \\
& =\frac{1}{h^{N} s^{N+1}} \int \prod_{a=1}^{N} \mathrm{~d} \mu\left(p_{a}\right) \exp \left(-s \frac{m \sigma}{2} \varphi_{N}\right) .
\end{aligned}
$$

Using expressions (4.11) and (4.19), (4.20) we find

$$
Z_{N}(s, \beta)=\frac{1}{h^{N} s^{N+1}} z\left(\beta, M_{2}\right)^{2} z\left(\beta, M_{1}\right)^{N-2} .
$$

where

$$
M_{1}^{2}=m^{2}+2 s \frac{m \sigma}{\beta}, \quad M_{2}^{2}=m^{2}+s \frac{m \sigma}{\beta},
$$

and

$$
z(\beta, m)=\int_{0}^{\infty} \mathrm{d} p \exp \left[-\frac{1}{2} \beta\left(p+\frac{m^{2}}{p}\right)\right]=2 m K_{1}(\beta m) .
$$

Next, we consider the grand partition function

$$
Z(\beta, s \lambda)=\sum_{N} \lambda^{N} Z_{N}(\beta, s)
$$

The summation over $N$ is performed immediately giving

$$
Z(\beta, s, \lambda)=\frac{z^{2}\left(\beta M_{2}\right)}{z^{2}\left(\beta M_{1}\right)} \frac{h}{s h-\lambda z\left(\beta M_{1}\right)} .
$$

The asymptotic behaviour of function $Z(\beta, A)$ is determined by the singularity points of function (4.27) which lie on the real axis for variable $s$. In our case there exists only one such a point, $s^{\prime}$, that is the solution to the equation

$$
s^{\prime} h=\lambda z\left(\beta M_{1}\left(s^{\prime}\right)\right) .
$$

Specifically, we have for the pressure $P=\beta^{-1} s^{\prime}$ [18]. Using (4.24) and (3.18) we obtain an expression for the chemical potential in terms of $\beta$ and $P$ :

$$
\mu(\beta, P)=-\frac{1}{\beta} \ln \frac{z(\beta M)}{h \beta P},
$$

where

$$
M^{2}=m^{2}+2 m \sigma P .
$$

The standard thermodynamical relation [16]

$$
\mathrm{d} \mu=-\tilde{s} \mathrm{~d} T+v \mathrm{~d} P
$$


where $\tilde{s}=S / N$ and $v=A / N$, allows one to obtain the equation of state

$$
v=\frac{\partial \mu}{\partial P}=\frac{1}{\beta P}+\frac{m \sigma}{M} \frac{K_{0}(\beta M)}{K_{1}(\beta M)} .
$$

It is convenient to represent this equation in the form:

$$
v=\frac{1}{\beta P}+\sigma \delta(\beta, P),
$$

where

$$
\delta(\beta, P)=\frac{m}{M} \frac{K_{0}(\beta M)}{K_{1}(\beta M)}
$$

From the inequality

$$
K_{n}(x)>K_{m}(x), \quad \text { if } \quad n>m,
$$

it follows that

$$
0<\delta(\beta, P)<1
$$

In the non-relativistic approximation, when $\beta m \rightarrow \infty, M \rightarrow m$, the asymptotical expression (3.11) shows that $\delta \rightarrow 1$, and we obtain a well-known Tonks equation [19]

$$
v=\frac{1}{\beta P}+\sigma .
$$

In the ultra-relativistic limit, when $\beta m \rightarrow 0$, we have $\delta \rightarrow 0$ and arrive at the state equation of the ideal gas. In the case of small $\sigma$ (that corresponds to the linear approximation in the interaction) we can replace function $\delta(\beta, P)$ by its value $\delta_{0}(\beta)$ at $\sigma \rightarrow 0$ :

$$
\delta_{0}=\frac{K_{0}(\beta m)}{K_{1}(\beta m)} .
$$

This quantity gets an interesting interpretation after accounting that the mean value of $p^{n}$ over the free-particle distribution defined by the Hamiltonian (4.2) is given by

$$
\left\langle p^{k}\right\rangle_{0}=\int_{0}^{\infty} \mathrm{d} p p^{k} \mathrm{e}^{-\beta H_{0}(p)} / \int_{0}^{\infty} \mathrm{d} p \mathrm{e}^{-\beta H_{0}(p)}=m^{k} \frac{K_{n+1}(\beta m)}{K_{1}(\beta m)} .
$$

Then $\delta_{0}(\beta)=m\left\langle p^{-1}\right\rangle_{0}$. Next, we can observe that in the front form of dynamics we have for free particles $p=m \gamma$, where $\gamma^{-1}=\sqrt{1-2 v}$ corresponds to the Lorentz radical $\sqrt{1-v^{2}}$. Therefore, in the linear approximation the state equation (4.33) can be considered as a result of taking into account the well-known Lorentz spatial contraction $\sigma \rightarrow \sigma\left\langle\gamma^{-1}\right\rangle_{0}$ in the non-relativistic Tonks equation (4.37).

From the equation of state (4.32) we get immediately

$$
\frac{\partial v}{\partial P}=-\frac{1}{\beta P^{2}}-\beta\left(\frac{m \sigma}{M}\right)^{2}\left[1-\frac{K_{0}^{2}(\beta M)}{K_{1}^{2}(\beta M)}\right] .
$$


Then inequality (4.35) shows that the condition of thermodynamical stability

$$
\left(\frac{\partial v}{\partial P}\right)_{T}<0
$$

is valid for equation (4.32) identically. Thus, the system does not have any phase transition.

In a similar manner we may obtain explicit expressions for other thermodynamical functions. For example, for entropy we have

$$
\begin{aligned}
\tilde{s} & =-\left(\frac{\partial \mu}{\partial T}\right)_{P}=k \beta^{2} \frac{\partial \mu}{\partial \beta} \\
& =k\left\{\ln \frac{2 M K_{1}(\beta M)}{h \beta P}+2+\beta M \frac{K_{0}(\beta M)}{K_{1}(\beta M)}\right\} ;
\end{aligned}
$$

the energy density is determined by

$$
\tilde{u}=\mu+T \tilde{s}-P v=\frac{1}{\beta}+\frac{m^{2}+m \sigma P}{M} \frac{K_{0}(\beta M)}{K_{1}(\beta M)} .
$$

Specific heat $c_{P}$ can be directly obtained from (4.42) giving

$$
\begin{aligned}
c_{P} & =T\left(\frac{\partial \tilde{s}}{\partial T}\right)_{P}=-\beta\left(\frac{\partial \tilde{s}}{\partial \beta}\right) \\
& =k\left\{2+(\beta M)^{2}\left[1-\frac{K_{0}(\beta M)^{2}}{K_{1}(\beta M)^{2}}-\frac{K_{0}(\beta M)}{\beta M K_{1}(\beta M)^{2}}\right]\right\} .
\end{aligned}
$$

Therefore, we have an exactly solvable example of a non-trivial particle interaction in the relativistic statistical mechanics.

\section{Weakly relativistic corrections to the thermodynamics of an interacting particle system}

Here we shall consider a system of $N$ point-like particles with pairwise interactions at the first post-Newtonian approximation. Let the non-relativistic interaction potential has the form:

$$
U^{(0)}=\sum_{a<b} \sum_{a b} ; \quad u_{a b}=u\left(r_{a b}\right), \quad r_{a b} \equiv\left|\mathbf{r}_{a b}\right| \equiv\left|\mathbf{x}_{\mathbf{a}}-\mathbf{x}_{b}\right| .
$$

In that case the general form of the first post-Newtonian Lagrangian function is given by [1]:

$$
\begin{aligned}
& L=\sum_{a}\left(\frac{m_{a} v_{a}^{2}}{2}+\frac{m_{a} v_{a}^{4}}{8 c^{2}}\right)-U^{(0)} \\
& +\frac{1}{2 c^{2}} \sum_{a<b} \sum_{\left(\mathbf{v}_{a} \cdot \mathbf{v}_{b} u_{a b}-\left(\mathbf{r}_{a b} \cdot \mathbf{v}_{a}\right)\left(\mathbf{r}_{a b} \cdot \mathbf{v}_{b}\right) \frac{1}{r_{a b}} \frac{d u_{a b}}{d r_{a b}}\right)+c^{-2} \Phi}
\end{aligned}
$$


with an arbitrary Galilei-invariant function $\Phi$. We choose this function in the form which is determined in terms of the non-relativistic potential $u(r)$ :

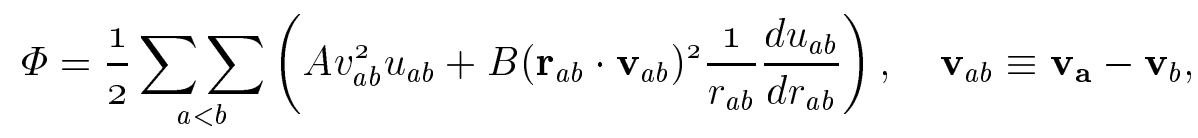

with two arbitrary numerical coefficients, $A$ and $B$. This structure of the postNewtonian Lagrangian is sufficient to cover a wide class of interactions including those following from various field-theoretical considerations. For example, the values

$$
A=\sigma^{2}-1, \quad B=0
$$

correspond to the interactions mediated by linear relativistic fields of spin $\sigma$. Particularly, $\sigma=1$ and $u(r) \sim r^{-1}$ give the famous Darwin's Lagrangian for electromagnetic interactions. It is remarkable that expression (5.2) under (5.3) was derived by Breit [20] as far back as 1937 with the aid of simple symmetry treatment.

For the system of identical particles the Hamiltonian which follows from the above Lagrangian has the form:

$$
H=H^{(0)}+c^{-2} H^{(1)}+O\left(c^{-4}\right),
$$

where $H^{(0)}$ is a non-relativistic Hamiltonian,

$$
H^{(0)}=\sum_{a} \frac{p_{a}^{2}}{2 m}+U^{(0)}
$$

and

$$
\begin{aligned}
& H^{(1)}=-\sum_{a} \frac{p_{a}^{4}}{8 m^{3}}-\frac{1}{2 m^{2}} \sum_{a<b} \sum_{<}\left\{\left[(1-2 A) \mathbf{p}_{a} \cdot \mathbf{p}_{b}+A\left(p_{a}^{2}+p_{b}^{2}\right)\right] u_{a b}\right. \\
& \left.-\left[(1+2 B)\left(\mathbf{r}_{a b} \cdot \mathbf{p}_{a}\right)\left(\mathbf{r}_{a b} \cdot \mathbf{p}_{b}\right)-B\left[\left(\mathbf{r}_{a b} \cdot \mathbf{p}_{a}\right)^{2}+\left(\mathbf{r}_{a b} \cdot \mathbf{p}_{b}\right)^{2}\right]\right] \frac{1}{r_{a b}} \frac{d u_{a b}}{d r_{a b}}\right\} .
\end{aligned}
$$

The canonical variables $\left(\mathbf{x}_{a}, \mathbf{p}_{a}\right)$ are connected with the Lagrangian $\left(\mathbf{x}_{a}, \mathbf{v}_{a}\right)$ by the standard Legendre transformation

$$
\mathbf{p}_{a}=\frac{\partial L}{\partial \mathbf{v}_{a}}
$$

considered to the order $c^{-2}$. Inserting Hamiltonian (5.5) into expression (3.3) for the classical partition function and expanding it to the $c^{-2}$ terms, we get

$$
Z_{N}=\frac{1}{h^{3 N} N !} \int \mathrm{e}^{-\beta H^{(0)}}\left(1-\beta c^{-2} H^{(1)}\right) \prod_{a} \mathrm{~d}^{3} x_{a} \mathrm{~d}^{3} p_{a} \equiv Z_{N}^{(0)}+c^{-2} Z_{N}^{(1)},
$$

where $Z_{N}^{0}$ is a non-relativistic partition function

$$
Z_{N}^{0}=Z_{N}^{(0) i d} Q
$$


$Q$ being a non-relativistic configuration integral

$$
Q=V^{-N} \int \mathrm{e}^{-\beta U^{(0)}}\left(1-\beta c^{-2} H^{(1)}\right) \prod_{a} \mathrm{~d}^{3} x_{a} .
$$

The straightforward calculation gives first-order corrections to the non-relativistic partition function (5.10):

$$
Z_{N}^{(1)}=Z_{N}^{(0)} \frac{R}{\beta m} ; \quad Z_{N}=Z_{N}^{(0)}\left(1+\frac{R}{\beta m c^{2}}\right),
$$

where $R$ is defined in terms of the configuration integral (5.11),

$$
R=\frac{15}{8} N-\frac{3}{Q}\left(A \beta \frac{\partial Q}{\partial \beta}+B V \frac{\partial Q}{\partial V}\right)
$$

In the absence of interaction, when $Q=1$, the obtained expression agrees with equation (3.13).

All thermodynamical properties of the system may be deduced from the free energy:

$$
F=-\beta^{-1} \ln Z=F^{(0)}-\frac{R}{\beta^{2} m c^{2}},
$$

where the corresponding non-relativistic expression is given by

$$
F^{(0)}=-\beta^{-1} \ln \left[\left(V \Lambda^{-3}\right)^{N} \frac{Q}{N !}\right] .
$$

equation (5.14) can be rewritten in the form, which gives a first-order correction in terms of the non-relativistic free energy:

$$
F=F^{(0)}-\frac{3}{\beta m c^{2}}\left[\left(\frac{5}{8}-\frac{3 A}{2}+B\right) \frac{N}{\beta}+A\left(F^{(0)}+\beta \frac{\partial F^{(0)}}{\partial \beta}\right)+B V \frac{\partial F^{(0)}}{\partial V}\right] .
$$

This formula may be also useful in the obtaining of weakly relativistic corrections to various phenomenological non-relativistic results.

Let us consider corrections to the equation of state. In the non-relativistic limit it has the form:

$$
P=-\frac{\partial F^{(0)}}{\partial V} \equiv g^{(0)}(\beta, V) .
$$

Then (5.16) gives a weakly relativistic equation of state in the form

$$
P=g^{(0)}(\beta, V)-\frac{3}{\beta m c^{2}}\left((A+B) g^{(0)}+A \beta \frac{\partial g^{(0)}}{\partial \beta}+B V \frac{\partial g^{(0)}}{\partial V}\right),
$$

which is determined by the non-relativistic form (5.19) and the two constants, $A$ and $B$. 
As an example we can consider a well-known Van der Waals equation

$$
P=\frac{N}{\beta(V-N b)}-\frac{N^{2} a}{V^{2}}
$$

with a pair of phenomenological constants, $a$ and $b$. The corresponding weakly relativistic equation is

$$
P=\frac{N}{\beta(V-N b)}\left[1+\frac{3 N b B}{\beta m c^{2}(V-N b)}\right]-\frac{N^{2} a}{V^{2}}\left[1+\frac{3(B-A)}{\beta m c^{2}}\right] .
$$

Up to the first order in $c^{-2}$ it can be presented in the non-relativistic form (5.19) with constants $a$ and $b$ being replaced by the linear functions on temperature:

$$
a \mapsto a^{\prime}=a\left(1+3 \frac{B-A}{\beta m c^{2}}\right) ; \quad b \mapsto b^{\prime}=b\left(1+\frac{3 B}{\beta m c^{2}}\right)
$$

Corrections to other thermodynamical functions and a wider discussion can be found in $[15]$.

\section{References}

1. Gaida R. P. Quasirelativistic systems of interacting particles // Fiz. Elem. Chast. \& Atom. Yadra, 1982, vol. 13, issue 2, p. 427-493 (in Russian; transl. in English: Sov. J. Part. Nucl., 1982, vol. 13, p. 179).

2. Llosa J. (ed.) Relativistic Action at a Distance: Classical and Quantum Aspects. Proc. Workshop. Barcelona, 1981. Springer, 1982.

3. Longhi G., Lusanna L. (eds.) Proc. Workshop "Constraint's Theory and Relativistic Dynamics"(Florence, Italy, 1986). Singapore, World Sci. Publ., 1987.

4. Vladimirov Yu. S., Turygin A. Yu. Direct Interparticle Interaction Theory. Moskow, Energoatomizdat, 1986 (in Russian).

5. Ter Haar D., Wergeland H. Thermodynamics and statistical mechanics in the special theory of relativity // Phys. Reports, 1971, vol. 1, No 2, p. 31-54.

6. Gaida R. P., Tretyak V. I. Single-time form of the Fokker-type relativistic dynamics. I // Acta Phys. Pol. B, 1980, vol. 11, No 7, p. 509-522.

7. Gaida R. P., Kluchkovsky Yu. B., Tretyak V. I. Lagrangian classical relativistic mechanics of a system of directly interacting particles. I // Teor. Mat. Fiz. 1980, vol. 44, № 2, p. 194-208 (in Russian; transl. in English: Theor. Math. Phys., 1980, vol. 44, No 2, p. 687-697).

8. Gaida R. P., Kluchkovsky Yu. B., and Tretyak V. I. Forms of relativistic dynamics in the classical Lagrangian description of a system of particles // Teor. Mat. Fiz., 1983, vol. 55, № 1, p. 88-105 (in Russian; transl. in English: Theor. Math. Phys., 1983, vol. 55, No 1, p. 372-384).

9. Gaida R. P., Kluchkovsky Yu. B., and Tretyak V. I. Three-dimensional Lagrangian approach to the classical relativistic dynamics of directly interacting particles. - In: Constraint's Theory and Relativistic Dynamics, G. Longhi and L. Lusanna, eds. Singapore, World Scientific Publ., 1987, p. 210-241. 
10. Dirac P. A. M. Forms of relativistic dynamics // Rev. Mod. Phys., 1949, vol. 21, No 3, p.392-399.

11. Dirac P. A. M. Lectures on Quantum Mechanics. New York, Yeshiva Univ., 1964.

12. Pirani F. A. E., Robinson D. C., Shadwick W. T. Local Jet Bundle Formulation of Bäcklund Transformations. Reidel, Dordrecht, 1979.

13. Sudarshan E. C. G. and Mukunda N. Classical Dynamics: A Modern Perspective. New York, Wiley, 1974.

14. Sokolov S. N. and Tretyak V. I. Front form of relativistic Lagrangian dynamics in two-dimensional space-time and its relation to Hamiltonian description // Teor. Mat. Fiz., 1986, vol. 67, No 1, p. 102-114 (in Russian).

15. Tretyak V. Thermodynamical properties of classical weakly-relativistic gas // Physical Collection, Shevchenko Scientific Society in Lviv, 1993. Vol. 1, p. 182-196 (in Ukrainian).

16. Balesku R. Equilibrium and Nonequilibrium Statistical Mechanics. New York, Wiley, 1975.

17. Singh S. P., Hasan M. Thermodynamics of nonrelativistic free quark gas // Indian J. Theor. Phys., 1984, vol. 32, No 8, p. 209-218.

18. Zinovjev G. M., Shelest V. P. Model of phase transition between hadronic and quarkgluone matter. - In: Fundamental Interactions (Physics). Moskow, MGPI, 1984, p. 323 (in Russian).

19. Landsberg R. T. Problems in Thermodynamics and Statistical Physics. London, 1971.

20. Breit G. Approximately relativistic equations for nuclear particles // Phys. Rev., 1937, vol. 51, p. 248-262.

\section{Про релятивістичні моделі в рівноважній статистичній механіці}

\section{В.Третяк}

Інститут фізики конденсованих систем НАН України, 290011 м. Львів-11, вул. Свєнціцького, 1

Отримано 12 червня 1998 р.

Досліджуються релятивістичні ефекти у термодинамічних властивостях систем взаємодіючих частинок у рамах релятивістичної теорії прямої взаємодії у різних формах динаміки. У фронтальній формі релятивістичної динаміки сформульовано одновимірну модель газу твердих сфер, що має точний розв'язок; знайдено рівняння стану та термодинамічні потенціяли. Обговорено слабкорелятивістичні поправки до термодинамічних функцій розрідженого газу з близькосяжними взаємодіями на основі наближеної релятивістичної функції Гамільтона у миттєвій формі динаміки.

Ключові слова: релятивістична статистична механіка, форми динаміки, релятивістичний ідеальний газ, слабкорелятивістичні поправки

PACS: $05.70 . C$ 